\title{
Merging Geometric Phase and Plasmon Retardation Phase in Continuously Shaped Metasurfaces for Arbitrary Orbital Angular Momentum Generation
}

Yinghui Guo ${ }^{\ddagger}$, Mingbo $\mathrm{Pu}^{\ddagger}$, Zeyu Zhao ${ }^{\ddagger}$, Yanqin Wang, JinJin Jin, Ping Gao, Xiong Li, Xiaoliang Ma, and Xiangang Luo*

State Key Laboratory of Optical Technologies on Nano-Fabrication and Micro-Engineering, Institute of Optics and Electronics,

Chinese Academy of Sciences, P.O. Box 350, Chengdu 610209, China 


\section{Supplementary Section 1. Comparison of the phase retardations of light transmitting through isolate slit and periodic slits.}
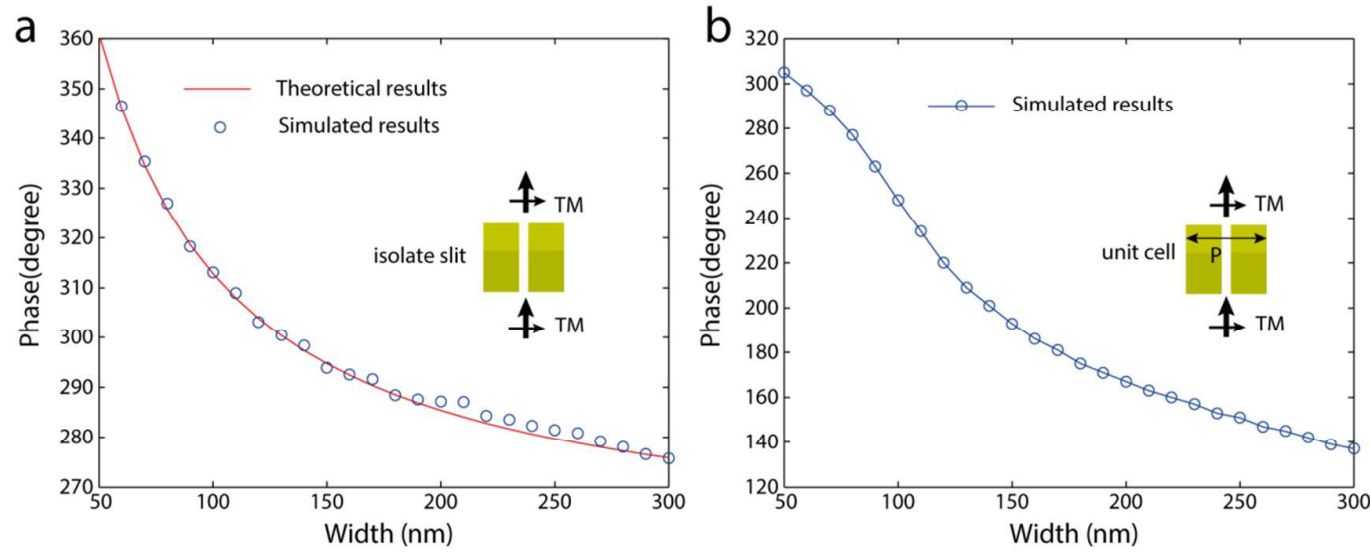

Figure S1. The phase retardations of light transmitting through (a) isolate slit and (b) periodic slits.

When isolate plasmonic slit is much smaller than the incident wavelength, only the fundamental propagation mode $\mathrm{TM}_{0}$ can exist in the structure, whose complex propagation constant $\beta$ satisfies the following dispersion relationship: ${ }^{1}$

$$
\tanh \left(\sqrt{\beta^{2}-k_{0}^{2}} \frac{w}{2}\right)=-\frac{\sqrt{\beta^{2}-k_{0}^{2} \varepsilon_{m}}}{\varepsilon_{m} \sqrt{\beta^{2}-k_{0}^{2}}}
$$

where $k_{0}$ is the wave vector of light in free space, $\varepsilon_{\mathrm{m}}=-18.36+0.479 i$ is the permittivity of the silver at $632.8 \mathrm{~nm}$, and $w$ is the slit width. The phase retardation of light transmitted through the plasmonic slit with a thickness of $d(450 \mathrm{~nm})$ is expressed as $\Phi=\beta d$. The calculated results are displayed in Figure S1a to compare with the simulation results from commercial software. Good agreement between them is found (Figure S1a). However, for periodic slits with a period of $P=$ $400 \mathrm{~nm}$, the phase retardation shown in Figure S1b is different with the case of isolate slit (Figure S1a). Obviously, when the slit width is changed, periodic slits obtain greater phase abrupt than isolate slit, which is welcome in wave-front engineereing. 
Supplementary Section 2. Numerically calculated diffraction patterns of continuously shaped metasurfaces with different numbers of the annular apertures.
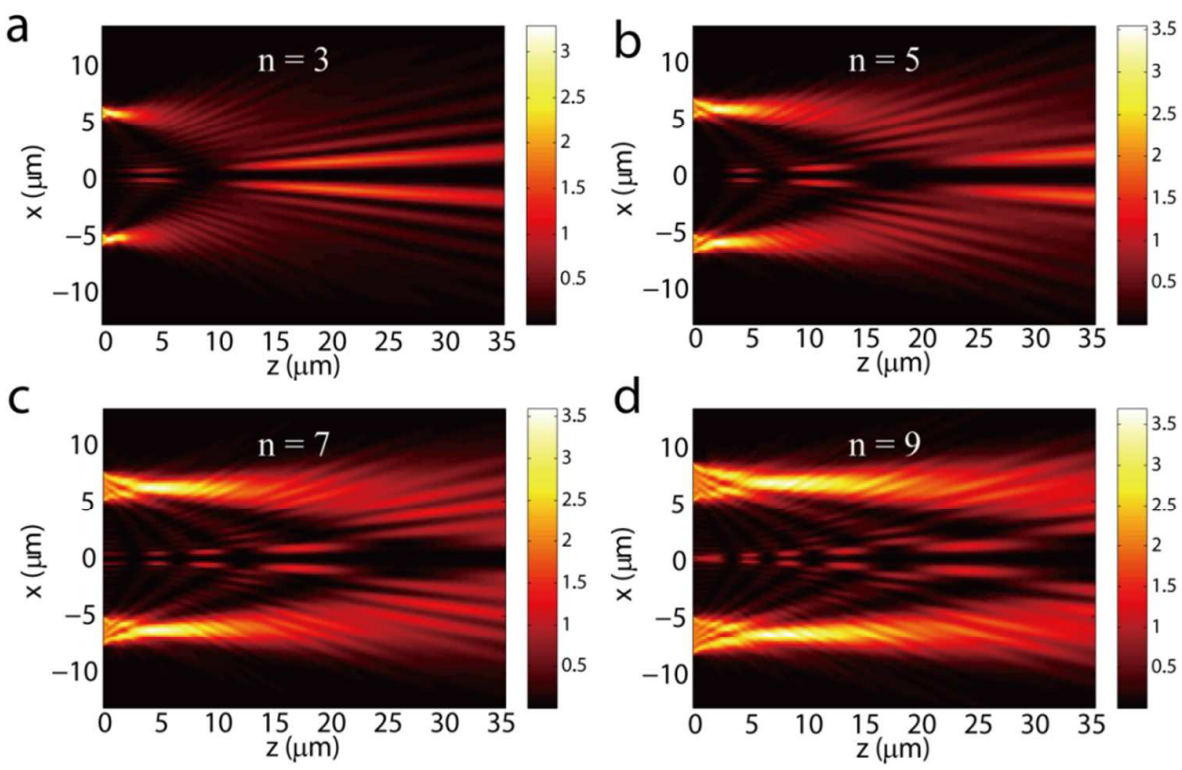

Figure S2. Numerically calculated longitudinal intensity distribution of cross-polarization light on the transmission side of the samples with different numbers of the annular apertures, whose radiuses keep to be $\mathrm{R}_{n}=5+(n-1) * P$. (a) $n=3$, (b) $n=5$, (c) $n=7$, and (d) $n=9$. The radiant intensity increases with the numbers of the annular apertures. Moreover, more annular apertures imply larger effective radiation aperture and better directionality. 
Supplementary Section 3. Numerically calculated diffraction patterns of continuously shaped metasurfaces with different annular radius.


Figure. S3. Numerically calculated intensity distribution of cross-polarization light on the transmission side of the samples with different radius and the numbers of the annular apertures keep to be $n=10$. (a) $\mathrm{R}_{n}=3+(n-1) * P$, (b) $\mathrm{R}_{n}=4+(n-1)^{*} P$, (c) $\mathrm{R}_{n}=5+(n-1)^{*} P$. The spot size of the hollow beam, defined as the dimmer of hollow beam, increases with the radius of the annular apertures. 
Supplementary Section 4. Design of elliptical apertures for generation of focusing sphere wave.
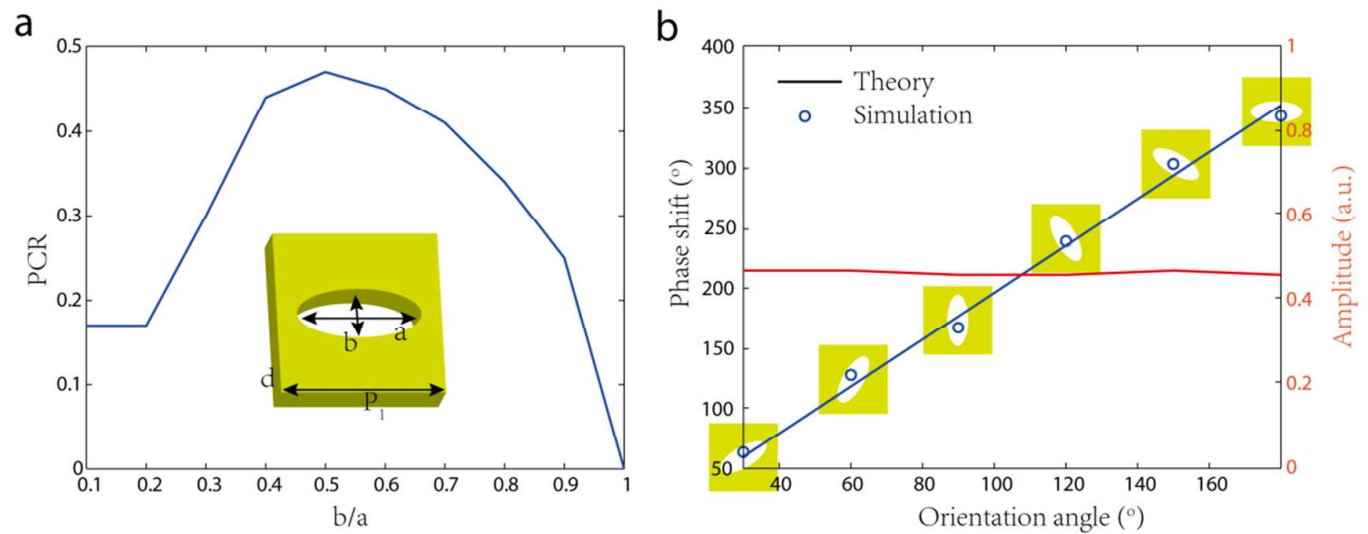

Figure. S4. (a) Polarization conversion ratio (PCR) versus with the axis ratio (b/a) of elliptical aperture, where $a$ and $b$ are the long axis and short axis of elliptical apertures. (b) Phase shift and amplitude as a function of orientation angle with LCP illumination at the wavelength of $632.8 \mathrm{~nm}$.

Full-wave simulations are performed within a unit cell by CST Microwave Studio with $\mathrm{P}_{1}=350$ $\mathrm{nm}, \mathrm{a}=300 \mathrm{~nm}$, and $\mathrm{d}=450 \mathrm{~nm}$. The maximum PCR is obtained when $\mathrm{b} / \mathrm{a}=0.5$ (i.e., $\mathrm{b}=150$ $\mathrm{nm}$ ), as indicated in Figure S4a. The phase shift shown in Figure S4b changes linearly with the orientation angle of elliptical nanohole and the amplitude is maintained well. A good agreement between the simulation and the theoretical prediction (According to the P-B phase principle, $\Phi=$ $2 \varphi$, where $\varphi$ is the orientation angle) is found. The slight deviation mainly stem from the angle dependence of light interaction between neighboring elliptical apertures. To produce a focusing spherical beam under the LCP illumination, the orientation angle of elliptical nanohole should satisfy:

$$
\varphi=\frac{\pi}{\lambda}\left(\sqrt{x^{2}+y^{2}+f^{2}}-f\right),
$$

where $f=20 \mu \mathrm{m}$ is the focus length of the spherical wave, $(x, y)$ are the coordinate of elliptical apertures covering an area of $9 \leq \sqrt{x^{2}+y^{2}} \leq 14.5$. 
Supplementary Section 5. Comparisons between experiment results and VAST results with the consideration of inclined illumination and residual polarization conserved term.

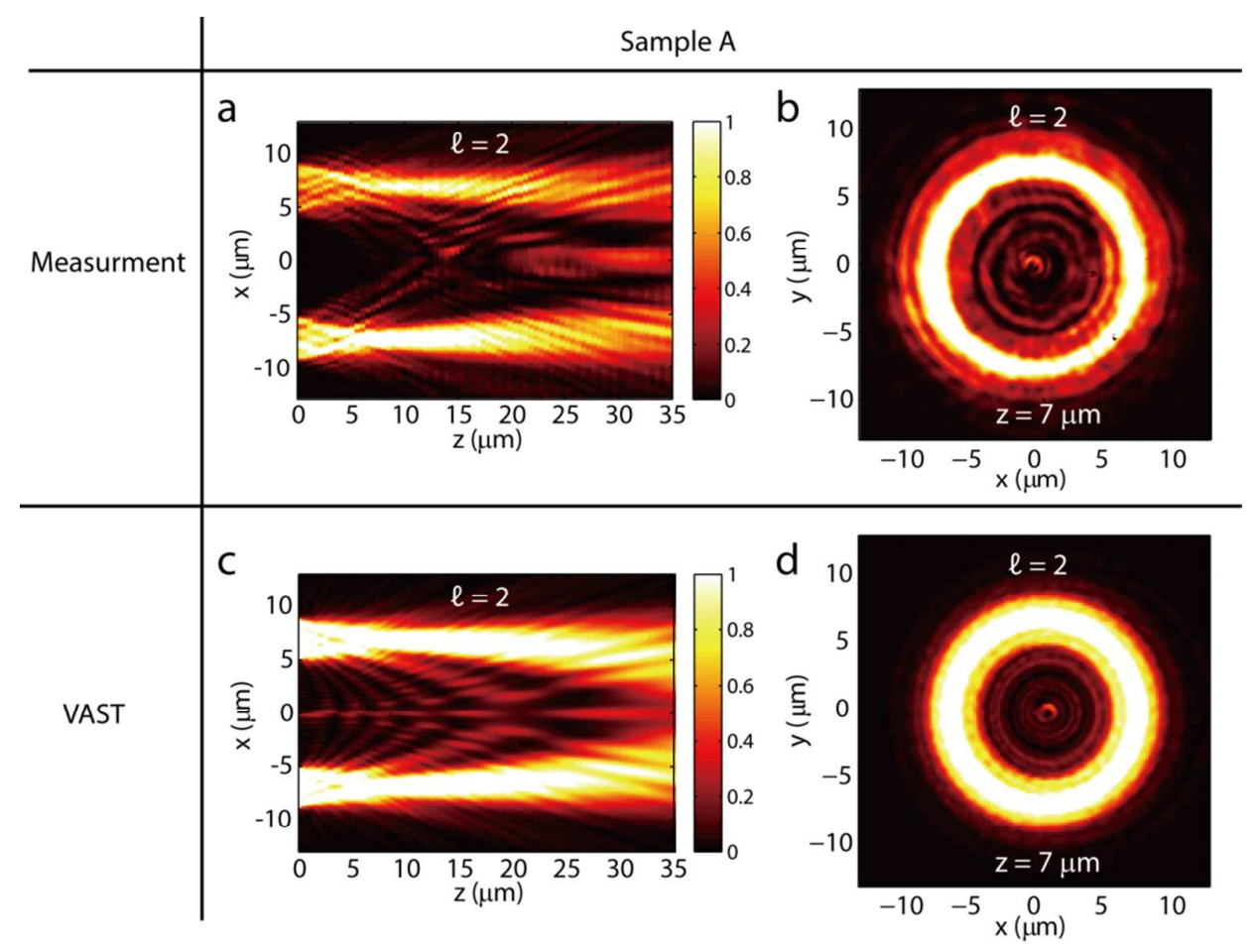

Figure. S5. (a) Measured longitudinal intensity distribution of polarization conversion term on the transmission side of the Sample A. (b) Measured transverse intensity profile at $z=7 \mu \mathrm{m}$ in (a). (c) (d) Corresponding vectorial angular spectrum theory (VAST) results with the considerations of inclined illumination about $5.7^{\circ}$ in the y direction and $23 \%$ polarization conserved term.

In this case, the VAST results in Figure S4c also exhibit on-axis patterns, especially at the positions after $\mathrm{z}=20 \mu \mathrm{m}$. Besides, the central part of the diffraction pattern shown in Figure S4(d) deviates from a perfect circle, just like the case in Figure S4(b). Better agreement between them can be found with proper inclined angle and residual intensity of polarization conserved term. It is no deny that the fabrication imperfections and material loss may also result the discrepancy. 
Supplementary Section 6. Simulated polarization conversion efficiency of the unit cells in continuously and discretely shaped metasurfaces.

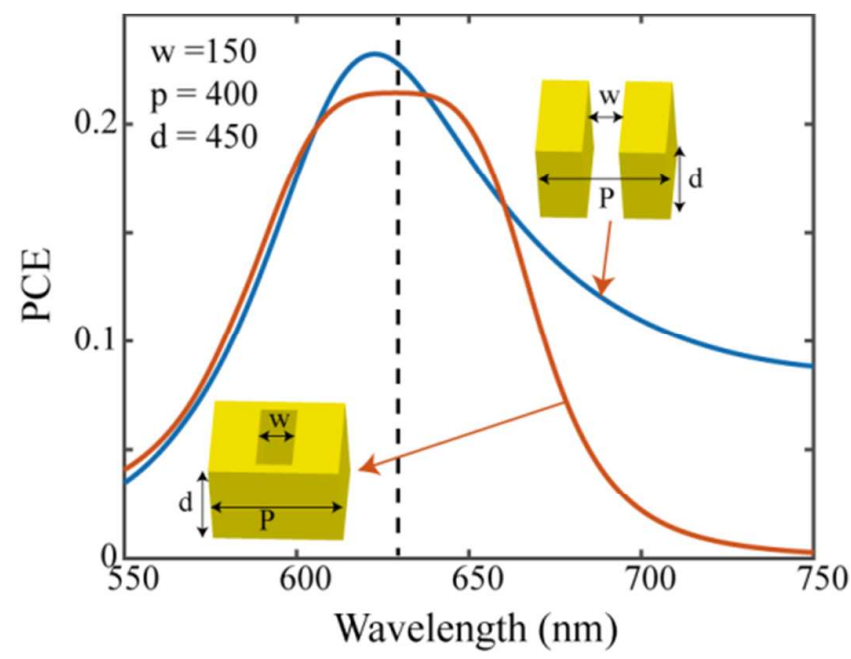

Figure. S6. Simulated polarization conversion efficiency (PCE) of the unit cells of continuously and discretely shaped metasurfaces, which have the same inner radius $(5 \mu \mathrm{m})$, ring number $(10)$, thickness $(450 \mathrm{~nm})$, period $(400 \mathrm{~nm})$, slit width $(150 \mathrm{~nm})$ and the unit cells of them exhibit approximate polarization conversion efficiency (i.e. $\sim 22 \%$ ).

\section{Acknowledgements}

We acknowledge the financial support by 973 Program of China under contract No. 2013CBA01700 and the National Natural Science Foundation of China under grants 61138002 and 61575201.

\section{AUTHOR INFORMATION}

\section{Corresponding Author}

*Email:1xg@ioe.ac.cn

Note: Y. Guo, M. Pu and Z. Zhao contribute equally to this work.

\section{References}

(1) Luo, X. Principles of Electromagnetic Waves in Metasurfaces. Sci. China-Phys. Mech. Astron. 2015, 58, 594201. 